\title{
High expression of microRNA-15b predicts a low risk of tumor recurrence following curative resection of hepatocellular carcinoma
}

\author{
GOH EUN CHUNG ${ }^{1}$, JUNG-HWAN YOON ${ }^{1}$, SUN JUNG MYUNG ${ }^{1}$, JEONG-HOON LEE ${ }^{1}$, SUNG-HEE LEE ${ }^{1}$, \\ SOO-MI LEE ${ }^{1}$, SEUNG-JUN KIM ${ }^{2}$, SEUNG YONG HWANG ${ }^{2}$, HYO-SUK LEE $^{1}$ and CHUNG YONG KIM ${ }^{1}$ \\ ${ }^{1}$ Department of Internal Medicine and Liver Research Institute, Seoul National University College of Medicine, Seoul; \\ ${ }^{2}$ Department of Biochemistry, Hanyang University and GenoCheck Co. Ltd., Gyeonggi-do, Korea
}

Received July 17, 2009; Accepted September 18, 2009

DOI: $10.3892 /$ or_00000612

\begin{abstract}
MicroRNAs (miRNA) have recently been implicated in carcinogenesis and tumor progression. Hepatocellular carcinoma (HCC) is well known for frequent relapses following curative resection. We attempted to identify the miRNAs associated with HCC recurrence. We analyzed miRNA expression profiles in 25 pairs of HCC and adjacent non-tumor liver tissues from HCC patients using miRNA microarray. Out of 449 miRNAs assayed, we identified seven miRNAs associated with HCC recurrence. In particular, the highest ranked miR-15b was negatively correlated with recurrence. MiR-15b inhibitor transfection increased HCC cell proliferation and inhibited TRAIL-induced apoptosis, while miR-15b precursor transfection decreased proliferation and enhanced apoptosis. Bcl-w was identified as a target molecule regulated by $\mathrm{miR}-15 \mathrm{~b}$. These results indicate that miR-15b expression in HCC tissues may predict a low risk of HCC recurrence. In addition, the modulation of miR-15b expression may be useful as an apoptosis-sensitizing strategy for HCC treatment.
\end{abstract}

\section{Introduction}

MicroRNAs (miRNA) are small interfering RNAs that regulate gene expression in a manner similar to transcription factors (1).

Correspondence to: Professor Jung-Hwan Yoon, Seoul National University Hospital, 28 Yungun-dong Chongno-gu, Seoul 110-744, Korea

E-mail: yoonjh@snu.ac.kr

Abbreviations: miRNA, microRNA; HCC, hepatocellular carcinoma; SAM, significance analysis of microarrays; MTS, 3,4(5-dimethylthiazol-2-yl)-5-(3-carboxymethoxyphenyl)-2-(4sulphenyl)- $2 H$-tetrazolium salt

Key words: miR-15b, hepatocellular carcinoma, recurrence, apoptosis, Bcl-w
Although the role of mammalian miRNAs still remains to be clarified, miRNAs have been implicated in a number of cellular processes including insulin secretion in the pancreas, differentiation of adipocytes and regulation of embryonic stem cell development. Abnormal expression of several miRNAs has been observed in several cancers including Burkitt's lymphoma, breast, liver, lung and colorectal cancer (2-5). In addition, differential expression of miRNAs has been found to be associated with postoperative survival as well as diagnostic and prognostic markers for lung cancer (6). Recently, it has been suggested that the abnormally expressed miRNAs, found in cancers, target transcripts of essential protein-coding genes involved in carcinogenesis (7).

Hepatocellular carcinoma (HCC) is one of the most common causes of death from cancer worldwide $(8,9)$. Resection or liver transplantation is the best treatment option for a potential cure. However, patients who undergo curative resection of HCC tumors frequently have tumor recurrence, and the postoperative 5-year survival is only $30-40 \%$ (10). Thus, clarification of the molecular mechanisms underlying HCC recurrence might help to identify therapeutic targets for the prevention of HCC recurrence. The objective of this study was to identify the miRNAs that are associated with HCC recurrence after curative resection.

\section{Materials and methods}

Samples. Twenty-five pairs of primary HCC and adjacent non-tumor liver tissues were evaluated in this study. These specimens were obtained from patients who underwent surgical resection at a tertiary hospital in Korea between 2001 and 2004. Their clinical characteristics are listed in Table I. HCC was diagnosed by histological confirmation. This study was approved by the Institutional Review Board of Seoul National University Hospital.

miRNA microarray and analysis. Total RNA was extracted from each sample using TRIzol reagent (Invitrogen, Carlsbad, CA, USA), were sent to Rosetta Genomics Corp. for RNA microarray experiments. Procedures were performed as described on the Web site of Rosetta (http://www.rosetta 
genomics.com). Briefly, $5 \mu \mathrm{g}$ of RNA from the sample was biotin-labeled during reverse transcription. Each sample was hybridized on a miRNA microarray slide consisting of 308 probes for human miRNAs. The values for the spots of each miRNA were background subtracted, normalized and subjected to further analysis. Normalization was performed by using per chip median normalization methods and variance stabilization and normalization (11). Differentially expressed miRNAs were identified by t-test analysis of the results of the significance analysis of microarrays (SAM). To filter out the miRNAs that exhibited the same expression levels across all samples, ANOVA was performed. Pairwise comparisons were performed on differentially expressed genes identified by ANOVA to determine how they differed from the non-tumor findings. For each pair of samples, a two-sample t-test was performed for every gene and multiplicity correction was applied using the step-up approach described by Benjamini and Hochberg (12). A false discovery rate of $5 \%$ was used.

Cell lines and transfection. Human hepatocellular carcinoma cell lines (SNU475, SNU761, Huh-7 and HepG2), one cholangio-carcinoma cell line (KMBC) and immortalized human hepatocytes (13) were used in this study. The stabilityenhanced miR-15b precursor (pre-miR; Ambion/Applied Biosystems, Foster, CA, USA) negative control and miR-15b inhibitor (Ambion/Applied Biosystems) were transfected using Lipofectamine 2000 reagent (Invitrogen) according to the manufacturer's instructions.

Real-time quantitative RT-PCR. RNA samples were reverse transcribed with miRNA-specific primers form TaqMan ${ }^{\circledR}$ MicroRNA Reverse Transcription Kit (Applied Biosystems, Foster City, CA). The reactions were performed using ABIpurchased primer probe sets with the ABI-Prism 7700 Sequence Detector System (Applied Biosystems). The expression level of the miRNA for each sample was calculated, reflecting the value of the threshold cycle $(\Delta \mathrm{Ct})$. The PCR conditions were $2 \mathrm{~min}$ at $50^{\circ} \mathrm{C}$ and $10 \mathrm{~min}$ at $95^{\circ} \mathrm{C}$, followed by 40 cycles of $95^{\circ} \mathrm{C}$ for $15 \mathrm{sec}, 58^{\circ} \mathrm{C}$ for $60 \mathrm{sec}$ and $95^{\circ} \mathrm{C}$ for $15 \mathrm{sec}$.

Cell proliferation. Cell proliferation was measured using CellTiter 96 Aqueous One Solution cell proliferation assays (Promega, Madison, WI, USA), based on the cellular conversion of the colorimetric reagent MTS [3,4-(5-dimethylthiazol-2-yl)-5-(3-carboxymethoxyphenyl)-2-(4-sulphenyl)$2 \mathrm{H}$-tetrazolium salt] into soluble formazan by dehydrogenase enzymes found only in metabolically active, proliferating cells. Following each treatment, $20 \mu \mathrm{l}$ of dye solution was added to each well of a 96-well plate and incubated for $3 \mathrm{~h}$. Subsequently, the absorbance was recorded using an ELISA plate reader at $490 \mathrm{~nm}$ (Molecular Devices, Sunnyvale, CA, USA).

Quantitation of apoptosis. Apoptosis was induced by TRAIL (100 ng/ml, Alexis Biochemicals, San Diego, CA, USA) and quantitated by assessing the characteristic nuclear change of apoptosis (i.e., chromatin condensation and nuclear fragmentation) using the nuclear binding dye DAPI [4', 6-
Table I. Clinical characteristics of patients at diagnosis.

\begin{tabular}{lr}
\hline Clinical variables & $\mathrm{n}(\%)$ \\
\hline Age $\left(\right.$ years) ${ }^{\mathrm{a}}$ & $61.5(36-77)$ \\
Gender & \\
Male & $22(88 \%)$ \\
Female & $3(12 \%)$ \\
Etiology & \\
HBV & $18(72 \%)$ \\
HCV & $2(8 \%)$ \\
NBNC & $5(20 \%)$ \\
Tumor size (cm) & $9(1.0-18.5)$ \\
CLIP stage & \\
0 & $9(36 \%)$ \\
1 & $10(40 \%)$ \\
$\geq 2$ & $6(24 \%)$ \\
UICC stage & \\
I & $6(24 \%)$ \\
II & $11(44 \%)$ \\
IIIA & $8(32 \%)$ \\
Recurrence & \\
Yes & $17.5(3.7-60.8)$ \\
No & $8.8(3.1-29.5)$ \\
Overall survival (months) & \\
Disease-free survival (months) & \\
& \\
&
\end{tabular}

${ }^{a}$ Median (range). HBV, hepatitis B virus; $\mathrm{HCV}$, hepatitis $\mathrm{C}$ virus and NBNC, non-B non-C.

diamidino-2-phenylindole dihydrochloride] (Sigma, St. Louis, MO, USA) and a fluorescence microscope (14).

Western blot analysis. The cells were lysed on ice with lysis buffer [50 mM Tris- $\mathrm{HCl}$ (pH 7.4); 1\% Nonidet P-40; $0.25 \%$ sodium deoxycholate; $150 \mathrm{mM} \mathrm{NaCl} ; 1 \mathrm{mM}$ EDTA; $1 \mathrm{mM}$ PMSF; $1 \mu \mathrm{g} / \mathrm{ml}$ aprotinin, leupeptin and pepstatin; $1 \mathrm{mM}$ $\mathrm{Na}_{3} \mathrm{VO}_{4}$; and $1 \mathrm{mM} \mathrm{NaF}$. The samples were resolved by sodium dodecyl sulfate polyacrylamide gel electrophoresis, transferred to nitrocellulose membranes, and blotted using the appropriate primary antibodies with peroxidase-conjugated secondary antibodies (Biosource International, Camarillo, CA, USA). The bound antibodies were visualized using a chemiluminescent substrate (ECL; Amersham, Arlington Heights, IL, USA) and exposed to film (X-Omat; Kodak, Hannover, Germany). The primary antibodies used were rabbit polyclonal Bcl-w antibody purchased from Santa Cruz Biotechnology Inc. (Santa Cruz, CA, USA); rabbit anticaspase 8 and rabbit anti-caspase 9 from Pharmingen (San Diego, CA, USA).

Statistical analysis. Cell proliferation and apoptosis experiments were repeated at least three times. Data are presented as the mean \pm standard deviation. The data were analyzed by ANOVA or the Student's t-test using the SPSS 
12.0 Windows version software. $\mathrm{P}<0.05$ was considered statistically significant.

\section{Results}

Differentially expressed miRNAs in HCC and non-tumor tissues. We selected the miRNAs that were measured as the smallest half of the data set sample. Among the 449 human miRNAs assayed, we identified 99 miRNAs expressed in human HCC tissues (Fig. 1). To find the miRNAs differentially expressed in the HCC tissues with respect to the corresponding non-tumor tissues, a statistical comparison between the two groups of samples was performed. The results showed 21 miRNAs that were differentially expressed in the HCC tissues (Table II). Based on these differentially expressed miRNAs, a supervised hierarchical cluster analysis was performed that generated a tree (Fig. 2A). Fourteen miRNAs including miR$15 \mathrm{~b}, \mathrm{miR}-105$ and miR-339 were up-regulated and eight were down-regulated in the HCC tissues.

Correlation between miRNA expression profiles and HCC recurrence. We next tried to identify the miRNAs associated with HCC recurrence by performing a K-nearest neighbor class prediction. This analysis yielded seven miRNAs that significantly separated the tumors according to their recurrence status with an overall accuracy of $90 \%$ (Fig. 2B). Among these, the highest ranked miR-15b was negatively correlated to HCC recurrence, whereas others including miR-34c and miR-361 were positively linked to tumor recurrence.

miR-15b function in HCC cells. To validate the microarray data, we performed RT-PCR of miR-15b in four different HCC cell lines (SNU475, SNU761, Huh-7 and HepG2), one cholangiocarcinoma cell line (KMBC) and one immortalized human hepatocyte cell line. As shown in Fig. 3A, miR-15b was expressed in the HCC cells, at the highest levels in the SNU475 cell line. The biological effect of miR-15b on cellular proliferation and apoptosis in the HCC cells was then evaluated by transfecting either the miR-15b inhibitor or precursor into the SNU475 cells. As shown in Fig. 3B, miR-15b inhibitor transfection enhanced cell proliferation, while miR-15b precursor transfection decreased cell proliferation. Transfection of the miR-15b inhibitor attenuated TRAIL-induced HCC cell apoptosis, whereas miR-15b precursor transfection augmented apoptosis (Fig. 4A and B). These observations collectively suggest that miR-15b overexpression in HCC cells may reduce cell proliferation and enhance cellular apoptosis, thereby reducing the risk of $\mathrm{HCC}$ recurrence following curative resection.

Down-regulation of $B c l-w$ protein expression by miR-15b. Given that the biological significance of miRNA regulation relies on their protein-coding gene targets, we analyzed the predicted targets of miR-15b. The analysis was performed using the three algorithms, miRanda, TargetScan and PicTar, commonly used to predict human miRNA gene targets. According to this analysis, we postulated that the gene for the BCL2-like 2, also known as Bcl-w, might be a target of miR-15b, since this protein is an anti-apoptotic Bcl-2 family member (15), and therefore its reduced expression, by miR-15b
Table II. Differentially expressed miRNAs in HCC tissues.

\begin{tabular}{|c|c|c|c|}
\hline miRNA & $\begin{array}{l}\text { SAM } \\
\text { scores }\end{array}$ & P-value & $\begin{array}{l}\text { SVM predictive } \\
\text { strength }\end{array}$ \\
\hline$h s a-m i R-15 b$ & 6.374815 & 0.000192 & 15.54 \\
\hline hsa-miR-105 & 4.238597 & 0.000383 & 14.11 \\
\hline hsa-miR-339 & 3.779922 & 0.000572 & 13.53 \\
\hline hsa-let-7d & 3.684254 & 0.000574 & 11.69 \\
\hline hsa-miR-107 & 3.632713 & 0.000764 & 9.945 \\
\hline hsa-miR-103 & 3.410522 & 0.001143 & 9.333 \\
\hline hsa-miR-210 & 3.216792 & 0.001892 & 9.021 \\
\hline hsa-miR-25 & 2.6496 & 0.0208 & 8.712 \\
\hline hsa-let-7a & 2.934883 & 0.003352 & 8.581 \\
\hline hsa-miR-93 & 2.770378 & 0.004878 & 8.372 \\
\hline hsa-miR-345 & 2.757579 & 0.004807 & 8.208 \\
\hline hsa-miR-30d & 2.683332 & 0.0161 & 8.04 \\
\hline hsa-miR-423 & 2.595492 & 0.005658 & 7.662 \\
\hline hsa-miR-320 & 2.7978 & 0.0148 & 7.503 \\
\hline$h s a-m i R-422 b$ & -2.91327 & 0.0055 & 10.23 \\
\hline hsa-miR-22 & -3.49419 & 0.005325 & 10.63 \\
\hline hsa-miR-497 & -3.72324 & 0.004619 & 11.15 \\
\hline hsa-miR-195 & -4.01715 & 0.00324 & 12.3 \\
\hline hsa-miR-199a* & -4.48924 & 0.000574 & 13.7 \\
\hline hsa-miR-199a & -5.1628 & 0.000378 & 14.84 \\
\hline hsa-miR-130a & -5.79953 & 0.000192 & 22.3 \\
\hline
\end{tabular}

miRNA, microRNA; SAM, significance analysis of microarrays and SVM, support vector machines.

overexpression, may be responsible for enhanced TRAILinduced HCC cell apoptosis. Indeed, we observed decreased expression of Bcl-w following miR-15b precursor transfection, which was reversed by inhibitor transfection (Fig. 4C). These data indicate that miR-15b overexpression in HCC cells may reduce Bcl-w expression, thereby leading to enhancement of cellular apoptosis.

\section{Discussion}

The principal finding of this study was the association of miRNAs with HCC recurrence: miR-15b expression in HCC tissues may predict a low risk of tumor recurrence following curative resection. Moreover, the results of the present study also suggest that the modulation of miR-15b expression may be therapeutically useful as an apoptosis sensitizing strategy.

miRNAs have recently been implicated in human carcinogenesis. Their expression profiles may reflect tumor characteristics, and they may be useful prognostic markers for cancers (16). The results of this study showed that 21 miRNAs were differentially regulated in HCC tissues when compared to non-tumor liver tissues. In particular, 14 miRNAs were up-regulated and seven were down-regulated in the HCC tissue. In a previous analysis of miRNA expression patterns in HCC tissues (4), eight miRNAs were found to be differentially regulated in HCC tissues. Among these, three miRNAs including miR-199a, miR-199a* and miR-195 were also found to be differentially expressed in our study. Taking into 


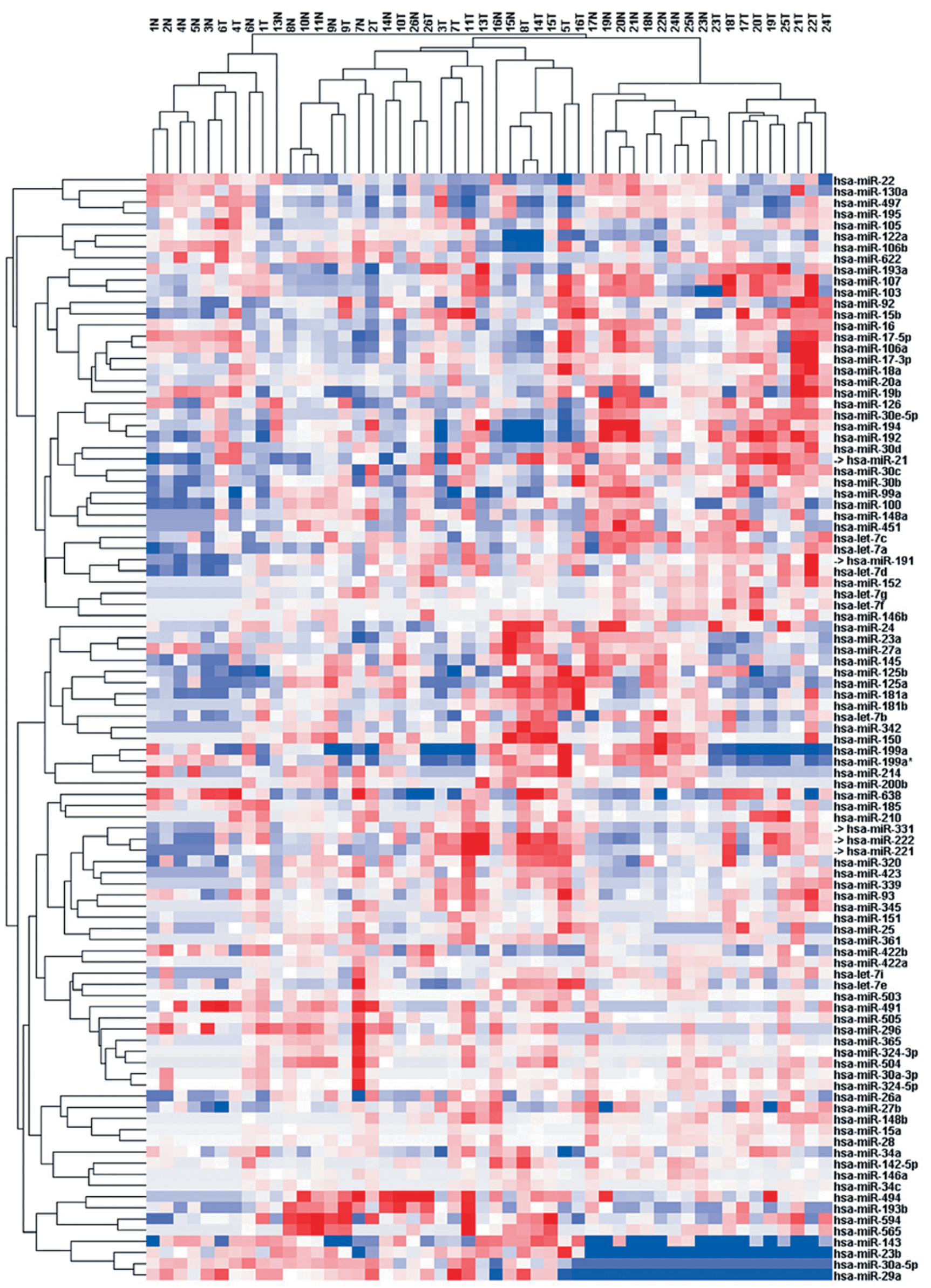

Figure 1. Cluster analysis of $50 \mathrm{HCC}$ samples and non-tumor liver tissues. miRNAs were included in the tree when their expression level (background-subtracted intensity) was higher than the threshold value (369) in at least 50\% of the samples. A total number of 99 miRNAs were retained for clustering. Arrays were median-centered and normalized using GeneSpring GX 7.3.1. Average linkage clustering was performed by the Pearson correlation metric. 


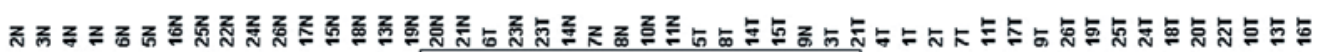

A

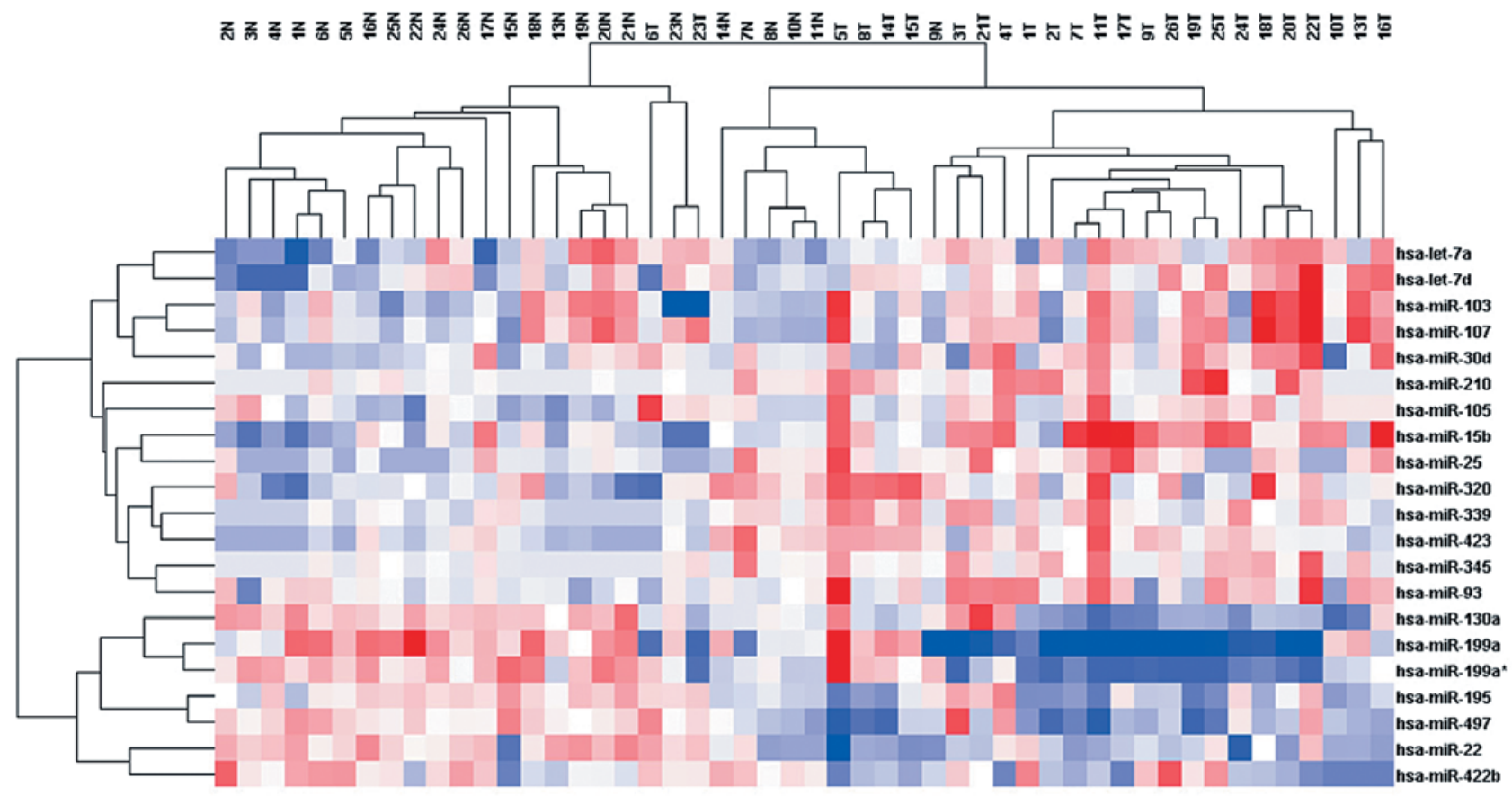

B

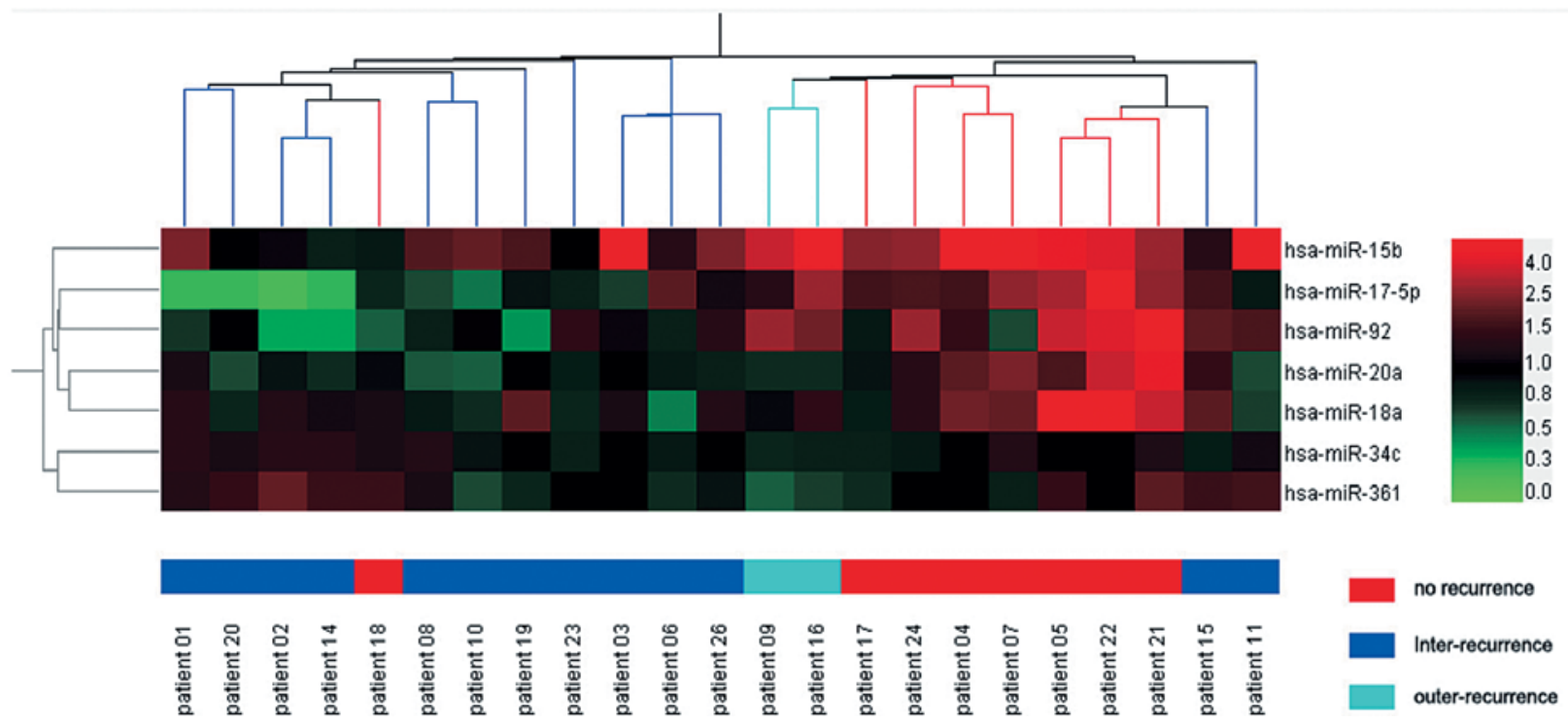

Figure 2. (A) miRNA expression signature in $25 \mathrm{HCC}$ patients. Twenty-one differentially expressed miRNAs were identified by the FDR procedure in the one-way ANOVA. The false discovery rate of $5 \%$ was used. (B) Cluster analysis of the 23 samples in the recurrence-clinical data. Seven miRNAs were identified using the Fisher's exact test within class. Arrays were median-centered and normalized using GeneSpring GX 7.3.1. Average linkage clustering was performed by the Pearson correlation metric.

consideration the different methods and etiologies in different study cohorts, it appears likely that these miRNAs participate in hepatocarcinogenesis and/or HCC progression.

Among the 99 miRNAs expressed in HCC tissues in our study, we additionally found that seven miRNAs may significantly differentiate the tumors according to their risk of recurrence following curative resection with an overall accuracy of $90 \%$. Among the seven miRNAs, miR-34c and miR-361 were positively linked to tumor recurrence. However, for lung cancer the outcome is different; miR-34c was repressed in human lung cancers and transfection of miR-34c significantly repressed lung cancer cell growth (17). One possible explanation for these findings is that the same miRNA can participate in different pathways, having varied effects on cell growth, survival and proliferation that depend on the cell type and pattern of gene expression (7). For example, miR-15a and miR-16-1 are down-regulated in CLL patients, but the same miRNAs are overexpressed in endocrine pancreatic tumors of neuroectodermal origin $(18,19)$.

Among the recurrence-related miRNAs identified in our study, the highest ranked miR-15b was negatively related to HCC recurrence, suggesting that it may potentially act as a tumor suppressor gene. Our functional studies additionally showed that the transfection of the miR-15b inhibitor in human HCC cells increased cell proliferation, while the miR-15b precursor transfection decreased proliferation. A recent study indicated that miR-15b might regulate cell cycle progression in glioma cells by targeting cell cycle-related molecules; 
A

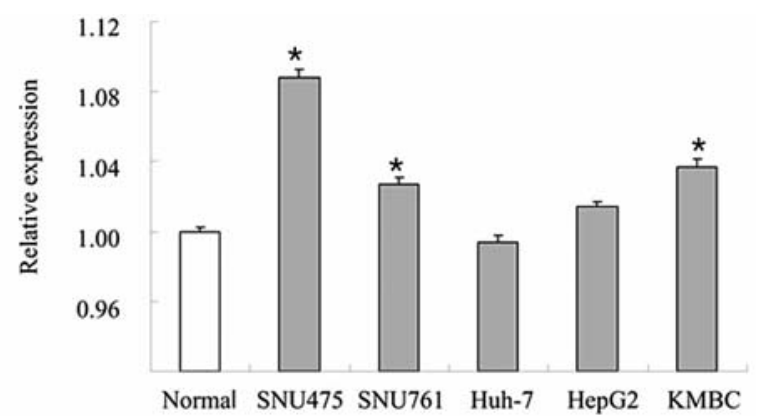

B

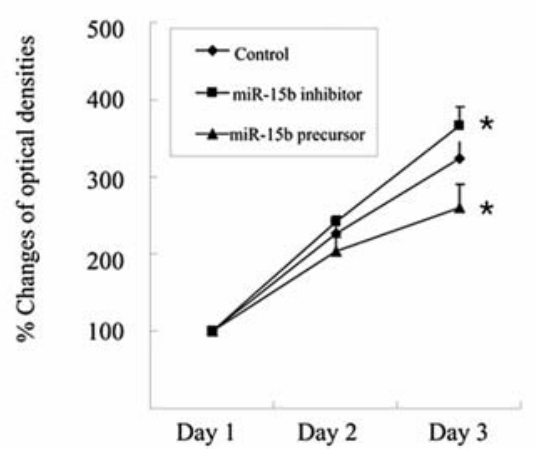

Figure 3. (A) miR-15b expression in the HCC cell lines. The miRNAs were isolated from four different HCC cell lines (SNU475, SNU761, Huh-7 and HepG2), one cholangiocarcinoma cell line (KMBC) and one immortalized human hepatocyte cell line. Quantitative real-time PCR for miR-15b was performed using a TaqMan microRNA assay kit. Quantitative data representing the relative expression $($ mean $+\mathrm{SD})$ from triplicate experiments are presented in the bar graph. ${ }^{*} \mathrm{P}<0.05$ compared to immortalized hepatocyte. (B) $\mathrm{miR}-15 \mathrm{~b}$ in HCC cell proliferation. SNU475 cells cultured in 96-well plates were transfected with the miR-15b inhibitor, miR-15b precursor or control. At each indicated point in time, an MTS assay was performed according to the manufacturer's instructions. ${ }^{*} \mathrm{P}<0.05$ compared to control. overexpression of miR-15b resulted in cell cycle arrest at the G0/G1 phase while suppression of miR-15b expression resulted in a decrease of cell populations in $\mathrm{G} 0 / \mathrm{G} 1$ and a corresponding increase of cell populations in the $\mathrm{S}$ phase (20). Therefore, it is most likely that the reduced HCC cell proliferation following miR-15b precursor transfection in our study resulted from miR-15b-induced modulation of cell cycle-related molecule expression.

In addition, our findings showed that miR-15b inhibitor transfection attenuated TRAIL-induced apoptosis, while miR-15b precursor transfection enhanced apoptosis. MiR-15a and miR-16, often deleted or down-regulated in a majority of cases with B-cell CLL, have previously been shown to promote apoptosis by targeting Bcl-2 $(21,22)$. MiR-15b and miR-16 were also shown to modulate multidrug resistance by targeting Bcl-2 in human gastric cancer cells (23). However, in the present study, we could not find any evidence that Bcl-2 expression levels were modulated by miR-15b in the HCC cells (data not shown).

Next we attempted to find the putative target of miR-15b by three computational prediction tools and found that $\mathrm{Bcl}-\mathrm{W}$ protein expression may be down-regulated by miR-15b. Indeed, we confirmed the decreased expression of Bcl-w following miR-15b precursor transfection, which was reversed by inhibitor transfection. In addition to miR-15b, miR-122 may also down-regulate Bcl-w expression in HCC cells and this modulation of Bcl-w expression was implicated in hepatocarcinogenesis (24). Therefore, the results of this study indicate that miR-15b overexpression in HCC cells may reduce Bcl-w expression, thereby leading to enhancement of cellular apoptosis and reduction of recurrence risk following curative resection.
A

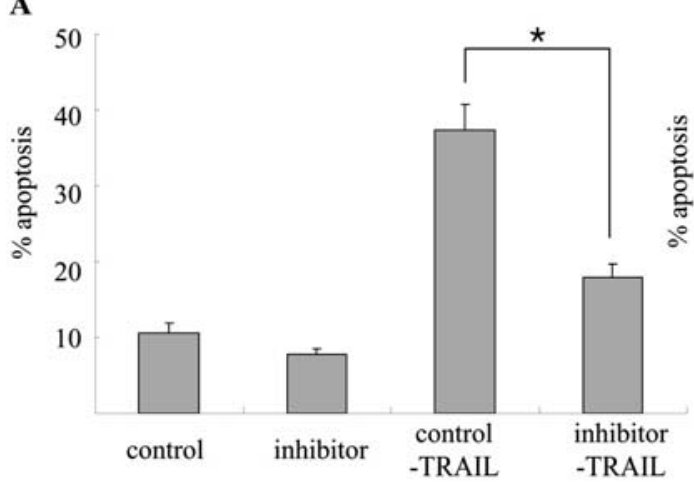

B

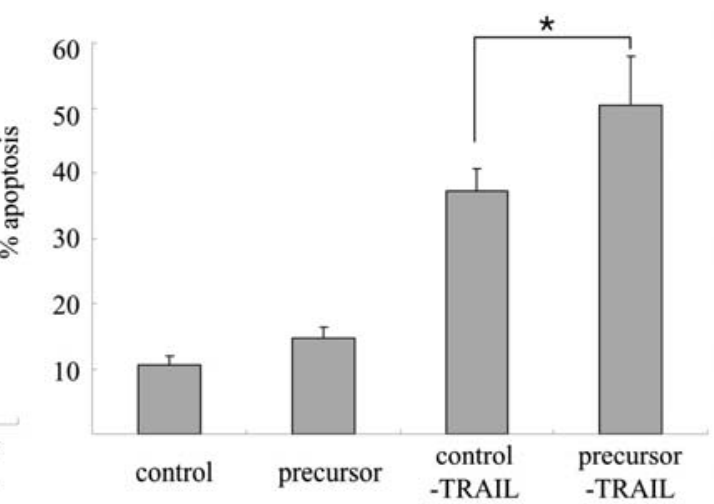

C

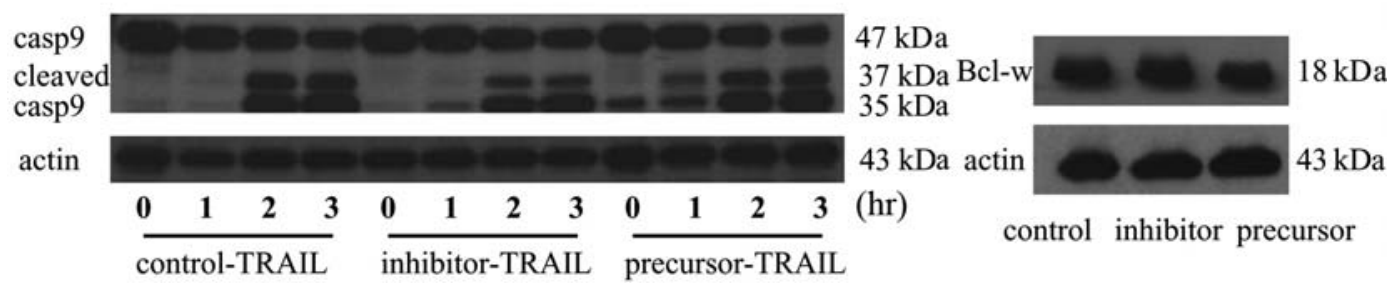

Figure 4. miR-15b in HCC cell apoptosis. SNU475 cells were transfected with miR-15b inhibitor, miR-15b precursor or control and then treated with TRAIL (100 ng/ml) for $3 \mathrm{~h}$. (A) Apoptosis was quantitated by fluorescence microscopy after DAPI staining. Data representing the mean +SD from triplicate experiments are presented in the bar graph. ${ }^{*} \mathrm{P}<0.05$. (B) Following TRAIL treatment, cells were lysed at each indicated point in time and immunoblot analyses were performed for caspase 9 and actin. (C) Western blot analysis of Bcl-w after transfection with miR-15b inhibitor, precursor or control. 
In conclusion, the findings of the present study demonstrate that miR-15b expression in HCC tissues may predict a low risk of tumor recurrence following curative resection, suggesting its prognostic significance. In addition, the modulation of miR-15b expression may be useful as an apoptosis-sensitizing strategy for HCC treatment.

\section{Acknowledgements}

This study was supported by the Seoul National University Hospital Research Fund (\#03-2006-019) and the Korean Foundation of Liver Research (2008) and the Korea Health 21 R\&D Project (\#0412-CR01-0704-0001).

\section{References}

1. Bartel DP: MicroRNAs: genomics, biogenesis, mechanism, and function. Cell 116: 281-297, 2004.

2. Lu J, Getz G, Miska EA, et al: MicroRNA expression profiles classify human cancers. Nature 435: 834-838, 2005

3. Iorio MV, Ferracin M, Liu CG, et al: MicroRNA gene expression deregulation in human breast cancer. Cancer Res 65: 7065-7070, 2005.

4. Murakami Y, Yasuda T, Saigo K, et al: Comprehensive analysis of microRNA expression patterns in hepatocellular carcinoma and non-tumorous tissues. Oncogene 25: 2537-2545, 2006.

5. Schepeler T, Reinert JT, Ostenfeld MS, et al: Diagnostic and prognostic microRNAs in stage II colon cancer. Cancer Res 68: 6416-6424, 2008.

6. Yanaihara N, Caplen N, Bowman E, et al: Unique microRNA molecular profiles in lung cancer diagnosis and prognosis. Cancer Cell 9: 189-198, 2006.

7. Calin GA and Croce CM: MicroRNA signatures in human cancers. Nat Rev Cancer 6: 857-866, 2006.

8. Seeff LB and Hoofnagle JH: Epidemiology of hepatocellular carcinoma in areas of low hepatitis B and hepatitis $\mathrm{C}$ endemicity. Oncogene 25: 3771-3777, 2006

9. Parkin DM, Bray F, Ferlay J and Pisani P: Global cancer statistics 2002. CA Cancer J Clin 55: 74-108, 2005.

10. El-Serag HB, Mason AC and Key C: Trends in survival of patients with hepatocellular carcinoma between 1977 and 1996 in the United States. Hepatology 33: 62-65, 2001.
11. Davison TS, Johnson CD and Andruss BF: Analyzing micro-RNA expression using microarrays. Methods Enzymol 411: 14-34, 2006.

12. Benjamini $\mathrm{Y}$ and Hochberg Y: Controlling the false discovery rate: A practical and powerful approach to multiple testing. JR Sta Soc Ser B 57: 289-300, 1995.

13. Yoon JH, Lee HS, Kim TH, Woo GH and Kim CY: Augmentation of urea-synthetic capacity by inhibition of nitric oxide synthesis in butyrate-induced differentiated human hepatocytes. FEBS Lett 474: 175-178, 2000.

14. Kwo P, Patel T, Bronk SF and Gores GJ: Nuclear serine protease activity contributes to bile acid-induced apoptosis in hepatocytes. Am J Physiol 268: 613-621, 1995.

15. Gibson L, Holmgreen SP, Huang DC, et al: bcl-w, a novel member of the bcl-2 family, promotes cell survival. Oncogene 13: 665-675, 1996.

16. Cho WC: OncomiRs: the discovery and progress of microRNAs in cancers. Mol Cancer 6: 60, 2007.

17. Liu X, Sempere LF, Galimberti F, et al: Uncovering growthsuppressive MicroRNAs in lung cancer. Clin Cancer Res 15: 1177-1183, 2009.

18. Volinia S, Calin GA, Liu CG, et al: A microRNA expression signature of human solid tumors defines cancer gene targets. Proc Natl Acad Sci USA 103: 2257-2261, 2006.

19. Roldo C, Missiaglia E, Hagan JP, et al: MicroRNA expression abnormalities in pancreatic endocrine and acinar tumors are associated with distinctive pathologic features and clinical behavior. J Clin Oncol 24: 4677-4684, 2006.

20. Xia H, Qi Y, Ng SS, et al: MicroRNA-15b regulates cell cycle progression by targeting cyclins in glioma cells. Biochem Biophys Res Commun 380: 205-210, 2009.

21. Cimmino A, Calin GA, Fabbri M, et al: miR-15 and miR-16 induce apoptosis by targeting BCL2. Proc Natl Acad Sci USA 102: 13944-13949, 2005.

22. Calin GA, Dumitru CD, Shimizu M, et al: Frequent deletions and down-regulation of micro- RNA genes miR15 and miR16 at $13 q 14$ in chronic lymphocytic leukemia. Proc Natl Acad Sci USA 99: 15524-15529, 2002.

23. Xia L, Zhang D, Du R, et al: miR-15b and miR-16 modulate multidrug resistance by targeting BCL2 in human gastric cancer cells. Int J Cancer 123: 372-379, 2008.

24. Lin CJ, Gong HY, Tseng HC, Wang WL and Wu JL: miR-122 targets an anti-apoptotic gene, Bcl-w, in human hepatocellular carcinoma cell lines. Biochem Biophys Res Commun 375: 315-320, 2008. 